\title{
Analysis of self-injection locked oscillators for motion sensing applications
}

\author{
Mabel Pontón, Almudena Suárez \\ University of Cantabria, Santander, Spain
}

\begin{abstract}
Self-injection locked oscillators have been recently proposed for motion sensing applications demonstrating a good experimental performance. Here a detailed investigation of the system dynamics is presented using a realistic model of the oscillator circuit under the injection of the reflected signal, which is phase modulated due to the target motion. The instability effects observed for some distance values are studied by means of a perturbation method and the results are validated through comparison with full circuit-level simulations and with measurements. The regular operation ranges are efficiently determined through a bifurcation analysis in terms of the distance to the target and the antenna gain. The modulation effect is analyzed with a reduced-order envelope transient formulation that copes with the accuracy problems associated with the small values of the modulation frequency. Very good agreement has been obtained with the experimental results.

Index Terms - Envelope-transient, self-injection locking, stability.
\end{abstract}

\section{INTRODUCTION}

Direct conversion Doppler radar systems have been proposed for motion sensing, with applications in person localization and breathing-rate measurement among other [1]. They are based on the transmission of a sinusoidal wave, such that the target, with zero net velocity, reflects this signal with its phase modulated proportionally to the time varying position, and the movement information is retrieved through phase demodulation. Recently, the possibility to implement the Doppler-radar system with a self-injection-locked oscillator (SILO) has been demonstrated [2]. The oscillator signal is transmitted to the target, reflected and received with a certain phase offset and phase modulation. The oscillator gets locked to the reflected signal, so it operates as a SILO. Although the valid performance of this kind of implementation has been experimentally demonstrated, the mathematical models are usually oversimplified, based on Adler's equation for synchronized oscillators [3], which should not be accurate since the overall system behaves like a free-running oscillator since the synchronizing signal is generated by the oscillator, which acts as both a transmitter and a receiver.

As any oscillatory system, the SILO operation ranges in terms of its sensitive parameters, such as the distance to the target or the antenna gain, will be delimited by qualitative stability changes or bifurcations [4]-[5], which have not been studied in any previous work to our knowledge. On the other hand, the phase modulation under a slow target movement will have a very low frequency, in the order of ten $\mathrm{Hz}$. This makes the simulation of the Doppler system difficult [1]-[2], since an envelope transient method should be used [6]-[8], able to consider the oscillation frequency as an unknown, which is not possible in commercial simulators.

The purpose of this work is to perform a detailed investigation of the Doppler radar based on SILO, with emphasis on stability analysis and bifurcation detection. It will be based on a reduced-order formulation, relying on a realistic model of the oscillator circuit. The formulation will be extended to consider the slow phase-modulation effects under the target movement. The results will be compared with harmonic-balance (HB) simulations (in the absence of any modulations) and with measurements.

\section{SELF-INJECTION LOCKED OSCILLATOR}

The SILO to be analyzed is sketched in Fig. 1(a). The oscillator is loaded with an antenna, which transmits the signal to the target, initially assumed to be motionless. The signal is reflected and received by the antenna with attenuation and phase shift effects, so the reflection coefficient for an antenna gain $G$ and distance to the target $d$ [Fig. 1(b)] can be calculated as [9]:

$$
\Gamma(G, d, \omega)=\sqrt{\frac{G^{2} \sigma \lambda^{2}}{(4 \pi)^{3} d^{4}}} e^{-j \omega \frac{2 d}{c}}=\frac{A_{o} G}{d^{2} \omega} e^{-j \omega \frac{2 d}{c}}
$$

where $\sigma$ is the radar cross section (assumed $0.5 \mathrm{~m}^{2}$ ), $c$ is the light velocity and $A_{o}$ is a global coefficient, introduced for compactness. The corresponding admittance, looking into the antenna terminals, will be: $Y=Y_{o}(1-\Gamma) /(1+\Gamma)$. For antenna gains between $5 \mathrm{~dB}$ and $12 \mathrm{~dB}$, frequency $f=\omega /(2 \pi)=2.4$ $\mathrm{GHz}$ and distance in the order of $0.5 \mathrm{~m}$, one obtains a magnitude of the reflection coefficient comprised between 0.028 and 0.142 . For larger distance, this magnitude will even be smaller. Thus, it will be possible to perform a Taylor series expansion of $Y$ about $\Gamma=0$, which provides: $Y=Y_{o}(1-2 \Gamma)$. After replacement of the original $Y_{o}$ load with $Y$ and substitution of (1), the steady-state oscillation equation, at the antenna connection terminals, is as follows:

$$
Y_{T}\left(V_{s}, \omega_{s}\right)=Y_{o s c}\left(V_{s}, \omega_{s}\right)-2 Y_{o} \frac{A_{o} G}{d^{2} \omega} e^{-j \omega_{s} \frac{2 d}{c}}=0
$$

where $Y_{\text {osc }}\left(V_{s}, \omega_{s}\right)$ is the oscillator admittance function. Assuming that the reflected signal only gives rise to a small 
increment of the steady-state oscillator solution, one can expand the admittance function $Y_{\text {osc }}\left(V_{s}, \omega_{s}\right)$ about the freerunning solution $V_{o}, \omega_{o}[10]$, which provides:

$Y_{T}\left(V_{s}, \omega_{s}\right) \cong Y_{v}\left(V_{s}-V_{o}\right)+Y_{\omega}\left(\omega_{s}-\omega_{o}\right)-2 Y_{o} \frac{A_{o} G}{d^{2} \omega_{s}} e^{-j \omega_{s} \frac{2 d}{c}}=0$

where $Y_{v}$ and $Y_{\omega}$ stand for the amplitude and frequency derivatives of the admittance function. These derivatives are calculated in a HB simulation of the standalone free-running oscillator, by applying finite differences to an auxiliary generator (AG) [4]-[5]. Note that the pure HB system constitutes an inner tier for this derivative calculation. Splitting (3) into real and imaginary parts:

$$
\begin{aligned}
& G_{v}\left(V_{s}-V_{o}\right)+G_{\omega}\left(\omega_{s}-\omega_{o}\right)-2 Y_{o} \frac{A_{o} G}{d^{2} \omega_{s}} \cos \left(\omega_{s} \frac{2 d}{c}\right)=0 \\
& B_{v}\left(V_{s}-V_{o}\right)+B_{\omega}\left(\omega_{s}-\omega_{o}\right)+2 Y_{o} \frac{A_{o} G}{d^{2} \omega_{s}} \sin \left(\omega_{s} \frac{2 d}{c}\right)=0
\end{aligned}
$$

The above system is composed of two real equations in two unknowns, $V_{s}, \omega_{s}$. The equations are linear in $V_{s}$ but nonlinear in $\omega_{s}$. The frequency fulfils the equation:

$$
\begin{aligned}
& \omega_{s}-\omega_{o}+2 Y_{o} \frac{A_{o} G}{\Delta d^{2} \omega_{s}} \cos \left(\omega_{s} \frac{2 d}{c}\right) B_{v}+ \\
& 2 Y_{o} \frac{A_{o} G}{\Delta d^{2} \omega_{s}} \sin \left(\omega_{s} \frac{2 d}{c}\right) G_{v}=0
\end{aligned}
$$

where $\Delta=G_{v} B_{\omega}-G_{\omega} B_{v}$. From inspection of (5), solutions will correspond to the intersection of a straight line of unit slope and a sinusoidal-variation function. For small $G / d^{2}$, only one intersection is possible. For larger values of $G / d^{2}$, several intersections may occur, giving rise to a multi-valued solution curve when varying $G$ or $d$. This has been verified solving equations (4) versus the distance $d$ for two different gain values: $G=3.5 \mathrm{~dB}$ and $G=10 \mathrm{~dB}$. Results are shown in Fig. 2. They have been compared with harmonic balance (HB) simulation using an AG, obtaining an excellent correspondence. Note that 8 harmonic terms have been considered in the HB simulation. For $G=10 \mathrm{~dB}$, the HB simulation experiences some convergence problems, as gathered from the incomplete sections of Fig. 2(b), due to its inability to pass through the turning points observed in the solution curve provided by (4). Measurement points have been superimposed in Fig. 2(a) and Fig. 2(b). The jumps in amplitude and frequency have been confirmed experimentally. Actually, Fig. 2(c) presents two spectra obtained for the same distance values, following a small hysteresis cycle.

The stability analysis will be based on the perturbation of equation (3) about the steady-state solution. The following function will be defined:

$$
F\left(\omega_{s}\right)=-2 Y_{o} \frac{A_{o} G}{d^{2} \omega_{s}} e^{-j \omega_{s} \frac{2 d}{c}}
$$

Under the perturbation effect, the oscillator amplitude and phase become $V_{s}+\delta v(t)$ and $\delta \varphi(t)$, respectively, and the frequency undergoes a complex increment $j \omega_{s}+s$. The increment $s$ will act like a time differentiator. Following a derivation similar to the one in [4]-[11] one obtains the following complex perturbation equation:

$$
Y_{v} \delta v(t)+\left(Y_{\omega}+\frac{\partial F\left(\omega_{s}\right)}{\partial \omega}\right)\left[-j \frac{\delta \dot{v}(t)}{V_{s}}+\delta \dot{\varphi}(t)\right]=0
$$

Splitting (7) into real and imaginary parts:

$$
\begin{aligned}
& G_{v} \delta v(t)+D_{\omega}^{i} \frac{\delta \dot{v}(t)}{V_{s}}+D_{\omega}^{r} \delta \dot{\varphi}(t)=0 \\
& B_{v} \delta v(t)-D_{\omega}^{r} \frac{\delta \dot{v}(t)}{V_{s}}+D_{\omega}^{i} \delta \dot{\varphi}(t)=0
\end{aligned}
$$

where the coefficient $D_{\omega}=Y_{\omega}+\frac{\partial F\left(\omega_{s}\right)}{\partial \omega}$ has been defined and the superindexes indicate real and imaginary parts. Equations in (8) can be arranged in a matrix form as follows:

$$
\left[\begin{array}{l}
\delta \dot{v}(t) \\
\delta \dot{\varphi}(t)
\end{array}\right]=-\left[\begin{array}{cc}
\frac{D_{\omega}^{i}}{V_{s}} & D_{\omega}^{r} \\
-\frac{D_{\omega}^{r}}{V_{s}} & D_{\omega}^{i}
\end{array}\right]^{-1}\left[\begin{array}{ll}
G_{v} & 0 \\
B_{v} & 0
\end{array}\right]\left[\begin{array}{l}
\delta v(t) \\
\delta \varphi(t)
\end{array}\right]
$$

The above linear time invariant system (9) has two eigenvalues. One is $\lambda_{1}=0$, associated with the full system autonomy. The other is:

$$
\lambda_{2}=\left(G_{v} D_{\omega}^{i}-B_{v} D_{\omega}^{r}\right) /\left[\left(D_{\omega}^{r}\right)^{2}+\left(D_{\omega}^{i}\right)^{2}\right]
$$

Replacing the expressions for the various elements in (10), one obtains that the turning points fulfill (4), together with the condition:

$$
\Delta+2 G_{v} Y_{o} \frac{A_{o} G}{d^{2} \omega_{s}} \sin \left(\omega_{s} \frac{2 d}{c}\right)+B_{v} 2 Y_{o} \frac{A_{o} G}{d^{2} \omega_{s}} \cos \left(\omega_{s} \frac{2 d}{c}\right)=0
$$

In order to choose the antenna gain and distance so as to avoid multi-valued responses (where undesired jumps between different solution sections would be observed), one may trace the turning point locus in the plane defined by the distance to the target and the antenna gain. This locus is given by the condition: $\lambda_{2}(d, G)=0$. Solving numerically for the $d$ and $G$ values that fulfill this condition, one obtains the plot in Fig. 3. Note that the loci will change with a different oscillator design, due to the associated change in the derivatives $Y_{v}, Y_{\omega}$. 


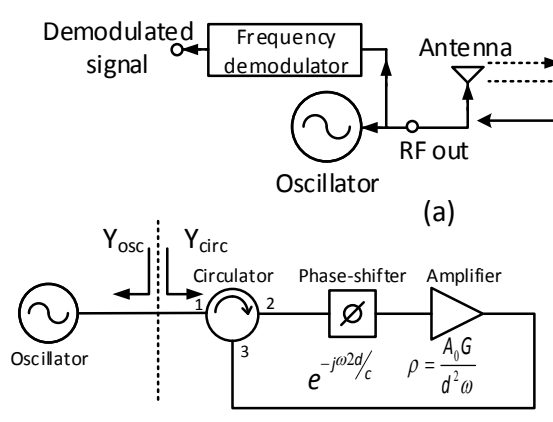

(b)
TARGET
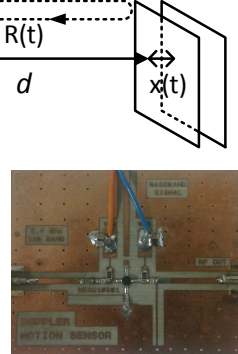

(c)
Fig. 1 Doppler radar system for motion sensing based on a SILO. (a) Operation with a single antenna. (b) Block diagram. (c) Photograph of the oscillator.

\section{ENVELOPE-TRANSIENT UNDER MODULATED CONDITIONS}

The case of a moving target will be considered. As in [1][2], the displacement $x(\mathrm{t})$ is assumed to be much smaller than the average distance to the target, that is, $x(\mathrm{t})<<d$. The total distance is now $2 d+2 x(t)$, which taking (2) into account will give rise to a phase modulation of the whole system. In [1], a typical target displacement is $5 \mathrm{~mm}$ at a frequency of $3 \mathrm{~Hz}$. Here an envelope transient formulation, based on [6], but adapted to our reduced-order model will be derived. In the presence of $x(\mathrm{t})$, the time-varying steady-state solution can be expressed:

$$
\bar{V}_{x}(t)=\left(V_{s}+\delta v(t)\right) e^{j \int \omega_{s}(t) d t+j \theta(t)}=\left(V_{s}+\delta v(t)\right) e^{j \varphi(t)}
$$

where the bar indicates that the variable is a phasor. The time variation $x(\mathrm{t})$ gives rise to a change in the carrier frequency, due to the system autonomy, and a time-varying phase $\theta(t)$. As shown in (12), it is possible to account for the two contributions in a single variable $\varphi(t)$, which provides:

$$
\begin{aligned}
& Y_{v}\left(V_{s}+\delta v(t)-V_{o}\right)+Y_{\omega}\left(\omega_{s}-j \frac{\delta \dot{v}(t)}{V_{s}}+\delta \dot{\varphi}(t)-\omega_{o}\right)+ \\
& \frac{\partial F\left(\omega_{s}\right)}{\partial \omega}\left[-j \frac{\delta \dot{v}(t)}{V_{s}}+\delta \dot{\varphi}(t)\right]+F\left(\omega_{s}, x(t)\right)=0
\end{aligned}
$$

where $F\left(\omega_{s}, x(t)\right)=-2 Y_{o} \frac{A_{0} G}{d^{2} \omega_{s}} e^{-j \omega_{s}\left(\frac{2 d+2 x(t)}{c}\right)}$. Expansion of this function in a Taylor series about $2 d$ allows one to eliminate the steady-state terms of (13). After splitting into real and imaginary parts one obtains:

$$
\begin{aligned}
& \left(B_{\omega}+F_{\omega}^{i}\right) \frac{\delta \dot{v}(t)}{V_{s}}+\left(G_{\omega}+F_{\omega}^{r}\right) \delta \dot{\varphi}(t)= \\
& -G_{v} \delta v(t)-x(t) 2 \omega_{s} F^{i}\left(\omega_{s}\right) / c-x^{2}(t) 2 \omega_{s}^{2} F^{r}\left(\omega_{s}\right) / 2 c^{2}- \\
& x(t)^{3} 4 \omega_{s}^{3} F^{i}\left(\omega_{s}\right) / 3 c^{3}+x^{4}(t) 2 \omega_{s}^{4} F^{r}\left(\omega_{s}\right) / 3 c^{4} \\
& \left(-G_{\omega}-F_{\omega}^{r}\right) \frac{\delta \dot{v}(t)}{V_{s}}+\left(B_{\omega}+F_{\omega}^{i}\right) \delta \dot{\varphi}(t)= \\
& -B_{v} \delta v(t)-x(t) 2 \omega_{s} F^{r}\left(\omega_{s}\right) / c+x^{2}(t) 2 \omega_{s}^{2} F^{i}\left(\omega_{s}\right) / 2 c^{2}- \\
& x(t)^{3} 4 \omega_{s}^{3} F^{i}\left(\omega_{s}\right) / 3 c^{3}-x^{4}(t) 2 \omega_{s}^{4} F^{i}\left(\omega_{s}\right) / 3 c^{4}
\end{aligned}
$$

This formulation has been applied to calculate the oscillator modulation effects due to the periodic motion of metal plate, with amplitude $1 \mathrm{~cm}$ and frequency $2.7 \mathrm{~Hz}$. In the experiment, the pseudo-periodic motion has been achieved with a motor. The signal is demodulated with a phase discriminator, including an amplifier. Fig. 4 compares the experimental demodulated signal with the theoretical one, obtained through demodulation of the oscillation signal in (14), with reasonable agreement.

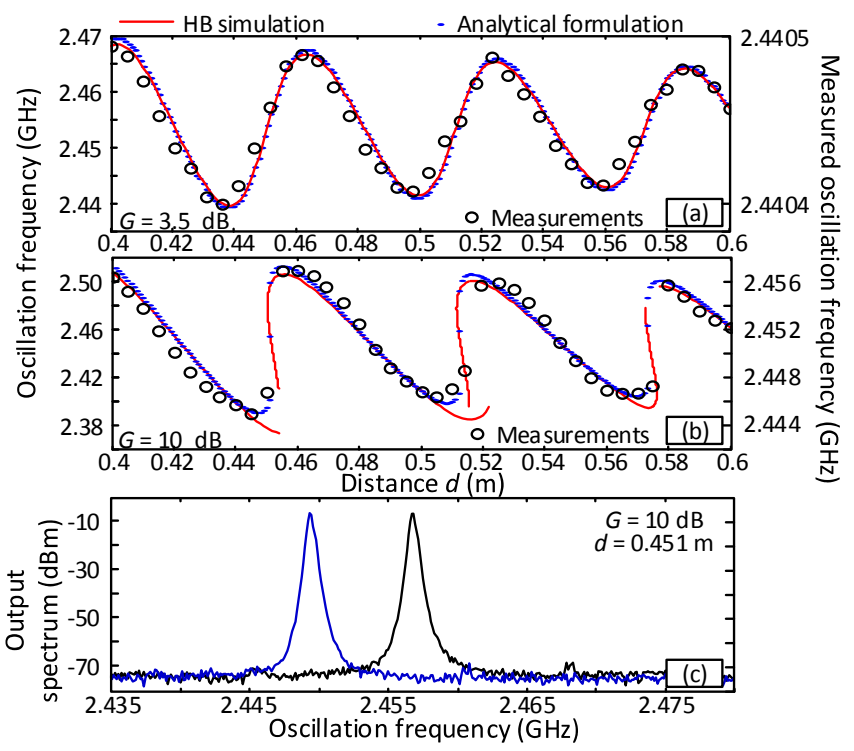

Fig. 2 Variation of the oscillation frequency with the distance to the target $d$ for two different values of antenna gain: (a) $G=3.5 \mathrm{~dB}$ and (b) $G=10 \mathrm{~dB}$. Results obtained with (4) are compared with those provided by HB with 8 harmonic terms.(c) Measured output spectra obtained for the same distance value $d=0.451 \mathrm{~m}$ and antenna gain $G=10 \mathrm{~dB}$. 


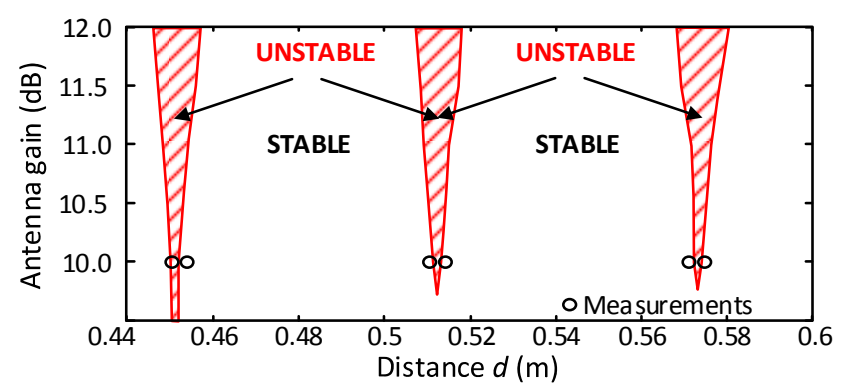

Fig. 3 Turning point loci delimiting the ranges of stable operation in the plane defined by the distance $d$ and the antenna gain $G$. Measurements are superimposed.

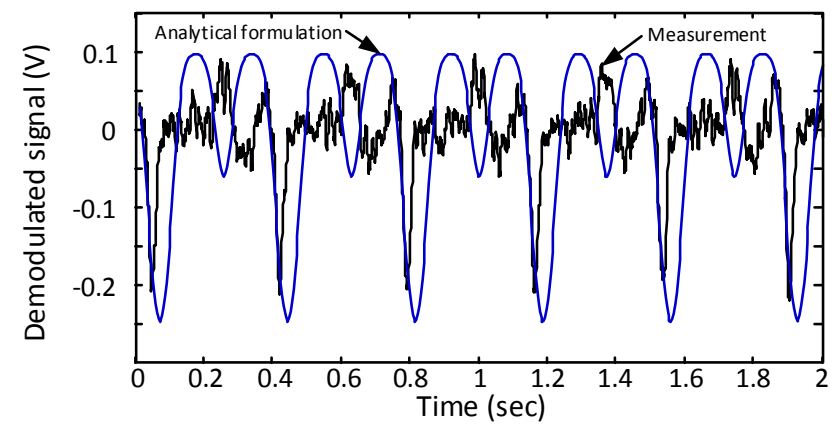

Fig. 4 Demodulated signal when the target undergoes a periodic motion with amplitude $1 \mathrm{~cm}$ and frequency $2.7 \mathrm{~Hz}$. The experimental demodulated signal is also represented.

\section{CONCLUSION}

An in-depth investigation of the dynamics of a Doppler radar based on a self-injection locked oscillators has been presented. The study is based on a semi-analytical formulation of the system, using an oscillator model extracted from a circuit-level simulation in HB. It includes the calculation of the solution curves, which can be multi-valued, the determination of the stability ranges and the analysis of the system response to a low frequency motion of the target, with an envelope-transient method.

\section{ACKNOWLEDGEMENT}

The authors would like to thank to Spanish Ministry of Economy and Competitiveness for their financial support under the research project TEC2014-60283-C3-1-R and Juan de la Cierva Research Program IJCI-2014-19141 and the Parliament of Cantabria for financial support under the project Cantabria Explora 12.JP02.64069.

\section{REFERENCES}

[1] A.D Droitcour, O. Boric-Lubecke and G.T.A. Kovacs, "Signalto-Noise Ratio in Doppler Radar System for Heart and Respiratory Rate Measurements," IEEE Trans. Microw. Theory Techn., vol.57, no.10, pp.2498-2507, Oct. 2009.

[2] F.K. Wang, T.S. Horng, K.C. Peng, J.K. Jau, J.Y Li and C.C Chen, "Single-Antenna Doppler Radars Using Self and Mutual
Injection Locking for Vital Sign Detection With Random Body Movement Cancellation," IEEE Trans. Microw. Theory Techn., vol.59, no.12, pp.3577-3587, Dec. 2011.

[3] R. Adler, "A study of locking phenomena in oscillators," Proc. IRE, vol.34, no.6, pp.351-357, Jun.1946.

[4] A. Suárez, Analysis and Design of Autonomous Microwave Circuits. Hoboken, NJ: Wiley IEEE Pres, 2009.

[5] A. Suárez, R. Quéré, Stability Analysis of Nonlinear Microwave Circuits, Artech-House Publishers, Boston, 2003.

[6] E. Ngoya and R. Larcheveque, "Envelope transient analysis: A new method for the transient and steady-state analysis of microwave communication circuits and systems," IEEE MTT-S Int. Microwave Symp., San Francisco, CA, 1996, pp. 1365-1368.

[7] E. de Cos, A. Suárez and S. Sancho, "Envelope transient analysis of self-oscillating mixers," IEEE Trans. Microwave Theory Techn., vol. 52, pp. 1090-1100, 2004.

[8] S. Sancho, A. Suárez and J. Chuan, "General envelope-transient formulation of phase-locked loops using three time scales," IEEE Trans. Microwave Theory Techn., vol. 52, pp. 1310-1320, 2004.

[9] B.J. Jang, S.H. Wi, J.G. Yook, M.Q. Lee, and K.J. Lee, "Wireless bio-radar sensor for heartbeat and respiration detection," Prog. Electromagn. Res., vol. 5, 149-168, 2008.

[10] A. Suárez and F. Ramírez, "Analysis of stabilization circuits for phase-noise reduction in microwave oscillators," IEEE Trans. Microw. Theory Techn., vol.53, no.9, pp.2743-2751, Sept. 2005.

[11] K. Kurokawa, "Some basic characteristics of broadband negative resistance oscillators," Bell Syst. Tech. J., vol. 48, pp. 1937 1955, Jul.-Aug., 1969. 